\title{
Singular Vortices on a Beta-Plane: A Brief Review and Recent Results
}

\author{
G. M. Reznik ${ }^{1, \bigotimes}$, S. V. Kravtsov ${ }^{1,2}$ \\ ${ }^{1}$ Shirshov Institute of Oceanology, Russian Academy of Sciences, Moscow, Russian Federation \\ ${ }^{2}$ University of Wisconsin, Milwaukee, USA \\ 凶greznikmd@yahoo.com
}

Purpose. This paper briefly reviews the theory of singular vortices (SV) on a beta-plane.

Methods and Results: The primary focus of the paper is on a long-term evolution of an individual SV: the governing equations and integrals of motion are given, the algorithm of numerical implementation of these equations for investigation of such an evolution is described, and the results of some numerical experiments are presented. It is shown that the vortex evolution consists of two stages. At an initial (quasi-linear) stage, the near-field radiation of Rossby waves by the vortex produces, near the vortex, a non-stationary secondary dipole - the beta-gyres - which forces the vortex to move (a cyclone drifts northwestward, an anticyclone - southwestward). At the next (nonlinear) stage, the far-field radiation of Rossby waves and self-interactions within the regular component of the motion become of importance. A singular cyclone (anticyclone) migrates slowly into the anticyclonic (cyclonic) beta-gyre; the SV and the beta-gyre form a compact vortex pair which continues to move northwestward (southwestward). As this process takes place, the cyclonic (anticyclonic) beta-gyre gradually drifts away from and ceases to affect the SV, while the SV starts to interact with the Rossby waves it radiated previously, which results in oscillations of its translation speed. The duration of the quasi-linear stage rapidly increases with an increasing intensity of the SV; for vortices of small or moderate intensity, this stage ends rapidly and gives way to the nonlinear stage. The first phenomenological description of the nonlinear stage of a singular monopole's evolution appeared in our recent work on the dynamics of the SV on a beta-plane.

Conclusions: The theory of singular vortices on a beta-plane developed here significantly broadens our understanding of the evolution and dynamics of localized geophysical vortices which play an important role in the large-scale circulation of the ocean and atmosphere.

Keywords: review of the theory of singular vortices on a beta-plane, Rossby waves

Acknowledgements: the work was carried out at financial support of the Ministry of Science and Higher Education of the Russian Federation (Agreement No. 05.616.21.0120, unique identifier RFMEFI61619X0120).

For citation: Reznik, G.M. and Kravtsov, S.V., 2020. Singular Vortices on a Beta-Plane: A Brief Review and Recent Results. Physical Oceanography, [e-journal] 27(6), pp. 659-676. doi:10.22449/1573-160X-2020-6-659-676

DOI: $10.22449 / 1573-160 \mathrm{X}-2020-6-659-676$

(C) G. M. Reznik, S. V. Kravtsov, 2020

(C) Physical Oceanography, 2020

\section{Introduction}

Discovery of oceanic synoptic vortices (see, e.g. [1] and references therein) provided a powerful impetus to the development of experimental and theoretical studies of vortex motion in a rotating fluid. Oceanic and atmospheric synoptic vortices possess an important ability to "self-propagate" relative to the rotating spherical Earth. For example, on a nonrotating sphere or on an f-plane with no mean currents, any radially symmetric vortex (hereafter, "monopole") remains unchanged and motionless; however, taking into account the $\beta$-effect, it begins to move along a certain trajectory, emitting Rossby waves. Mechanisms of this evolution have been studied by many authors analytically (see, e.g. [2-5]), ISSN 1573-160X PHYSICAL OCEANOGRAPHY VOL. 27 ISS. 6 (2020) 
numerically (see, e.g. [6-10]) and in laboratory experiments (see, e.g. [11, 12]). It was found that at an initial stage of the motion development, secondary dipolar vortices, the so-called beta-gyres, are generated near the vortex. This dipole "pushes" the vortex along its axis and, in turn, rotates as a whole in the direction determined by the vortex polarity. As a result (in the Northern Hemisphere), a cyclonic (anticyclonic) vortex moves to the northwest (southwest). However, it remained unclear whether the beta-gyres mechanism works until the vortex dissipates or other effects come into play with time, and if so, which ones and how they depend on the vortex parameters. These and some other questions are considered in the present paper, which is a short survey of the main results of the theory of singular vortices on a beta-plane, proposed in [2].

The work is organized as follows. In Section 2 the basic equations of the theory are presented, a brief discussion of the general properties of a system of singular vortices (SV) is given and equations and conservation integrals for an isolated SV are derived. In Section 3 the initial motion of an isolated SV and the development of beta-gyres are analyzed. Section 4 is devoted to the description of a numerical algorithm for computing the evolution of the systems of singular vortices. Section 5 presents recent results of numerical experiments on the long-time behavior of an isolated SV. A brief discussion of all these results is given in Section 6.

\section{Singular vortices. Problem statement}

Basic equations. The vortex dynamics will be studied within the framework of a 1.5-layer quasigeostrophic (QG) model on a beta-plane, described by the wellknown conservation equation for the QG potential vorticity (see, e.g., [1]) :

$$
\partial_{t}(\hat{\Omega}+\beta y)+J(\hat{\psi}, \hat{\Omega}+\beta y)=0, \quad \hat{\Omega}=\nabla^{2} \hat{\psi}-a^{2} \hat{\psi},
$$

where $\hat{\psi}=\hat{\psi}(x, y, t)$ is the streamfunction; $\hat{\Omega}$ is the relative vorticity; $\hat{\Omega}+\beta y$ is the potential vorticty; $a=R_{d}^{-1}, R_{d}$ is the Rossby scale; $\beta$ is the derivative of the Coriolis parameter with respect to latitude; $J$ is the Jacobian. The streamfunction $\hat{\psi}$ is the sum of a regular component $\psi$ and the singular component $\psi_{\mathrm{s}}$, the latter being represented by a superposition of $N$ Bessel vortices

$$
\begin{gathered}
\hat{\psi}=\psi+\psi_{s}, \quad \hat{\Omega}=\Omega+\Omega_{s}, \quad \Omega=\nabla^{2} \psi-a^{2} \psi, \quad \Omega_{s}=\nabla^{2} \psi_{s}-a^{2} \psi_{s}, \\
\psi_{s}=\sum_{n=1}^{N} \psi_{s}^{n}, \\
\psi_{s}^{n}=-\frac{A_{n}}{2 \pi} K_{0}\left[p_{n}\left|\mathbf{r}-\mathbf{r}_{n}(t)\right|\right], \\
\Omega_{s}=\sum_{n=1}^{N}\left[A_{n} \delta\left(x-x_{n}(t)\right) \delta\left(y-y_{n}(t)\right)+\left(p_{n}^{2}-a^{2}\right) \psi_{s}^{n}\right] .
\end{gathered}
$$

Here $\delta$ denotes the Dirac delta function; $K_{n}, I_{n}$ here and below - Bessel functions of order $n$; the intensities of the vortices $A_{n}$ and parameters $p_{n}$, which define the horizontal scales of the vortices, are given and assumed to be constant (see [2]); vortex trajectories $\mathbf{r}_{n}(t)$ are determined as a part of the solution.

Substitution of (2) into (1) gives, using (3), the following equations: 


$$
\begin{gathered}
\partial_{t} \Omega+J\left(\psi+\psi_{s}, \Omega+\beta y\right)+\sum_{n=1}^{N}\left(p_{n}^{2}-a^{2}\right) J\left(\psi+\psi_{s}^{n}+\dot{x}_{n} y-\dot{y}_{n} x, \psi_{s}^{n}\right)=0, \\
\dot{x}_{n}=-\partial_{y}\left(\psi+\psi_{s}^{n}\right)_{\mathbf{r}=\mathbf{r}_{n}}, \quad \dot{y}_{n}=\partial_{x}\left(\psi+\psi_{s}^{n}\right)_{\mathbf{r}=\mathbf{r}_{n}}, \quad \psi_{s}^{n}=\sum_{m \neq n}^{N} \psi_{s}^{m} .
\end{gathered}
$$

Equation (4) determines the evolution of the regular component $\psi$ and equations (5) - the SV trajectories. If the beta effect and the regular component are absent $(\beta=\psi=0)$ and $p_{n}=a$ (singular vorticies become the point ones), then (4) is satisfied identically, and (5) reduces to the well-known Hamiltonian system of ordinary differential equations for the coordinates of interacting vortices (see, e.g., [13]). If $\beta \neq 0$ and/or at the initial moment $\psi \neq 0$ and/or in a group of $N>1$ vortices, the scale of at least one of them is not equal to $R_{d}$, then the regular component $\psi$ is generated even if it is zero at the initial moment. To describe the motion in this case, one is to solve the complete continuous-discrete system (4), (5). A generalization of equations (2) - (5) to the case of a two-layer fluid is given in $[14,15]$.

Rossby solitons. If all of $p_{n}$ are equal to each other and $p_{n}=p \neq a$, then the SV intensities and positions can be selected in such a special way that the regular component vanishes and the vortices form a system moving uniformly and without changing shape along latitude with the velocity lying outside of the Rossby-waves phase-speed range $\left(-\beta R_{d}^{2}, 0\right)$. Each such system represents a singular Rossby soliton, and vice versa, each regular Rossby soliton is a superposition of the Bessel SVs (3b) with $p_{n}=p \neq a$, which are located in a finite inner domain of the soliton. The relationship between two-dimensional Rossby solitons and Bessel SVs (3b) is discussed in more detail in [2]. Singular Rossby solitons in a rotating barotropic spherical layer are presented in [16]. "Hybrid" Rossby solitons, consisting of a singular monopole and a regular component, were found in a two-layer model [15].

Governing equations and conservation integrals for an isolated SV. In what follows, we restrict our attention to the case of a single SV, when in (3) - (5) $N=1$ and the singular component of the streamfunction $\psi_{s}$ equals to

$$
\psi_{s}=-\frac{A}{2 \pi} K_{0}\left[p\left|\mathbf{r}-\mathbf{r}_{0}(t)\right|\right]
$$

Then equations (3) - (5) take the following form:

$$
\begin{gathered}
\partial_{t} \Omega+J\left(\psi_{s}, \Omega+\beta y\right)+J(\psi, \Omega+\beta y)+\left(p^{2}-a^{2}\right) J\left(\psi+\dot{x}_{0} y-\dot{y}_{0} x, \psi_{s}\right)=-K \nabla^{6} \psi, \\
\dot{x}_{0}=-\left.\partial_{y} \psi\right|_{\mathbf{r}=\mathbf{r}_{0}}, \\
\dot{y}_{0}=\left.\partial_{x} \psi\right|_{\mathbf{r}=\mathbf{r}_{0}}, \\
\left.\psi\right|_{t=0}=0 .
\end{gathered}
$$


Condition (8c) means that at the initial moment there is no regular field. In the remainder of the paper, the main part of the discussion deals with the numerical study of the SV; therefore, a scale-selective frictional formulation with a minimal hyperviscosity $K$ is added to the right side of Eq. (7) to ensure the stability of the numerical scheme presented below.

At $K=0$ the system (6) - (8) has several conservation integrals; at $K \neq 0$, these integrals are changing slowly with time. These integrals can be obtained by using an identity valid for any sufficiently smooth function $F(x, y)$ :

$$
\iint\left(\nabla^{2} F-p^{2} F\right) \psi_{s} d x d y=A F\left(x_{0}, y_{0}\right) ;
$$

here the area integral is taken over an unbounded plane. Multiplying (7) by the sum $\psi+\psi_{s}$ and integrating the resulting equation over the entire plane taking into account (8) and (9), one obtains the energy conservation equation

$$
\partial_{t}\left(E_{1}+E_{2}+E_{3}\right)=D_{1}+D_{2},
$$

where

$$
\begin{gathered}
E_{1}=\frac{1}{2} \iint\left((\nabla \psi)^{2}+a^{2} \psi^{2}\right) d x d y, \\
E_{2}=-\frac{1}{2}\left(p^{2}-a^{2}\right) \iint \psi \psi_{s} d x d y, \\
E_{3}=-\left.A \psi\right|_{\mathbf{r}=\mathbf{r}_{0}(t)} .
\end{gathered}
$$

Here $E_{1}$ is the energy of the regular component; the sum $E_{2}+E_{3}$ is the influx of energy from the singular vortex to the regular component; the terms

$$
\begin{array}{r}
D_{1}=-K \iint\left(\nabla\left(\nabla^{2} \psi\right)\right)^{2} d x d y, \\
D_{2}=K \iint \psi_{s} \nabla^{6} \psi d x d y
\end{array}
$$

are due to friction.

Further, multiplying (7) by $\nabla^{2} \psi-a^{2} \psi$ and again using (8) and (9), the enstrophy conservation law is obtained in the form

where

$$
\partial_{t}\left(S_{1}+S_{2}\right)=D_{3},
$$

$$
\begin{gathered}
S_{1}=\frac{1}{2} \iint\left[\left(\nabla^{2} \psi\right)^{2}+\left(p^{2}+a^{2}\right)(\nabla \psi)^{2}+p^{2} a^{2} \psi^{2}\right] d x d y, \\
S_{2}=-\beta A y_{0}, \\
D_{3}=-K \iint\left(\nabla^{4} \psi\right)^{2} d x d y+p^{2} D_{1} .
\end{gathered}
$$

A positive definite functional $S_{1}$ is a combination of the enstrophy and the energy associated with the regular flow.

Integration of (7) over the entire plane, taking into account (8c), gives the conservation of mass:

$$
\int \psi d x d y=0 \text {. }
$$


Conservation of the $x$ - and $y$-components of the momentum can be obtained by multiplying (7) by $x$ and $y$, respectively, with subsequent integration over the plane using (9), (12):

$$
\begin{gathered}
\int x \psi d x d y=\frac{A}{p^{2}}\left(\beta R_{d}^{2} t+x_{0}\right), \\
M_{1}-M_{2}=0, \\
M_{1}=\int y \psi d x d y, \\
M_{2}=\frac{A}{p^{2}} y_{0} .
\end{gathered}
$$

The equations (13a), (13b) mean that the gravity center of the entire vortex + wave system (i.e., of the field $\psi+\psi_{s}$ ) moves with velocity $-\beta R_{d}^{2}$ along the $x$-axis and does not shift in latitude. Integrals (10) - (13) can be generalized to the case of any number of singular vortices on a regular background current (see [2, 14]); it was shown in [14] that the conservation of energy (10) and enstrophy (11) guarantees, on an f-plane, the stability of any pair of interacting SVs with respect to arbitrary sufficiently small regular perturbations.

The original equation (1) and, consequently, (7) at $K=0$ postulate the Lagrangian conservation of potential vorticity (PV) $\Omega+\Omega_{s}+\beta y$ in a fluid parcel. By virtue of (8a) and (8b), the SV is associated with a given fluid parcel and the singular part of the vorticity $\Omega_{s}$ of this parcel remains constant (although infinite). Therefore, the regular part of the vorticity $q=\Omega+\beta y$ is conserved at the center of SV, i.e., knowing the SV position, $\left.\Omega\right|_{\mathbf{r}=\mathbf{r}_{0}}$ can be determined from

$$
q_{0}=\left.\Omega\right|_{\mathbf{r}=\mathbf{r}_{0}}+\beta y_{0}(t)=\beta y_{0}(0) .
$$

The above derivation is, strictly speaking, heuristic, but (14) can also be derived in a more rigorous way from (7), (8). The conservation integrals obtained above are used below to control the operation of a numerical scheme that allows one study the long-term evolution of an isolated SV.

\section{Beta-gyres and initial evolution of SV}

Let's suppose that $K=0$ and write (7) in moving coordinates

$$
x^{\prime}=x-x_{0}(t), y^{\prime}=y-y_{0}(t),
$$

associated with the vortex (omitting the primes):

$$
\begin{aligned}
& \partial_{t} \Omega+J\left(\psi_{s}, \Omega\right)+\beta \partial_{x} \psi_{s}+\beta \psi_{x}+J\left(\psi+\dot{x}_{0} y-\dot{y}_{0} x, \Omega\right)+ \\
& +\left(p^{2}-a^{2}\right) J\left(\psi+\dot{x}_{0} y-\dot{y}_{0} x, \psi_{s}\right)=0 .
\end{aligned}
$$

Owing to (8a) and (8b), the spatial derivatives of the streamfunction in moving coordinates $\psi+\dot{x}_{0} y-\dot{y}_{0} x$ vanish at the point of singularity $\mathbf{r}=\mathbf{r}_{0}(t)$. At an initial stage of the SV evolution, the regular streamfunction and vorticity are small and mainly localized in the vicinity of the SV; therefore, the fourth and fifth terms in 
(16) are small compared to the second and third terms and can be discarded. The resulting approximate equation is written as

$$
\partial_{t}(\Omega+\beta y)+J\left(\psi_{s}, \Omega+\beta y\right)+\left(p^{2}-a^{2}\right) J\left(\psi+\dot{x}_{0} y-\dot{y}_{0} x, \psi_{s}\right)=0
$$

Conditions (8) in moving coordinates associated with the vortex take the following form:

$$
\begin{aligned}
& \dot{x}_{0}=-\left.\partial_{y} \psi\right|_{\mathbf{r}=0}, \\
& \dot{y}_{0}=\left.\partial_{x} \psi\right|_{\mathbf{r}=0}, \\
& \left.\psi\right|_{t=0}=0 .
\end{aligned}
$$

The solution of problem (17) has the form

$$
\psi=\varphi_{s}(r, t) \sin \theta+\varphi_{c}(r, t) \cos \theta,
$$

where $r, \theta$ are polar coordinates centered at the location of singularity, and can easily be found in the case of a point vortex [2], when $p=a$ :

$$
\begin{gathered}
\Omega=\nabla^{2} \psi-a^{2} \psi=\beta r(\cos b t-1) \sin \theta-\beta r \sin b t \cos \theta, \quad b=A a^{2} K_{1}(a r) / 2 \pi a r, \\
\varphi_{s}=\beta I_{1}(a r) \int_{r}^{\infty} r^{2} K_{1}(a r)(1-\cos b t) d r+\beta K_{1}(a r) \int_{0}^{r} r^{2} I_{1}(a r)(1-\cos b t) d r, \\
\varphi_{c}=\beta I_{1}(a r) \int_{r}^{\infty} r^{2} K_{1}(a r) \sin b t d r+\beta K_{1}(a r) \int_{0}^{r} r^{2} I_{1}(a r) \sin b t d r .
\end{gathered}
$$

Substituting (19) into (8a), (8b), the components of the vortex velocity are found to be:

$$
\dot{x}_{0}=-\frac{1}{2} \beta R_{d}^{-1} \int_{0}^{\infty} r^{2} K_{1}(a r)(1-\cos b t) d r, \quad \dot{y}_{0}=\frac{1}{2} \beta R_{d}^{-1} \int_{0}^{\infty} r^{2} K_{1}(a r) \sin b t d r .
$$

Equations (18) - (20) describe the development of beta-gyres - a secondary circulation dipole in the vicinity of the SV, which induces the SV movement along the dipole axis. Knowing the SV's velocity, one can compute its trajectory (see below). In the case of a non-point SV, when $p \neq a$, the analytical expressions for the amplitudes $\varphi_{s}, \varphi_{c}$ in (18) are unknown and are to be determined numerically [10]. In the non-divergent case $a=0$ the problem (17) has an exact analytical solution for any $p$, as found in [17].

Evolution of the streamfunction $\psi$ at $p=a$, according to (18) - (20), is shown in Fig. 1; solutions for $p \neq a$ are qualitatively the same. The problem (17) is linear in $\psi$, and its solution $\psi$ is, in turn, linear in the beta-parameter, so the regime (17) will hereafter be referred to as the linear regime. The regular field $\psi$ here represents Rossby waves radiated by the singular vortex into the near zone; the absence of the term $\beta \psi_{x}$ in equation (17a) prevents the far-field propagation of Rossby waves. 

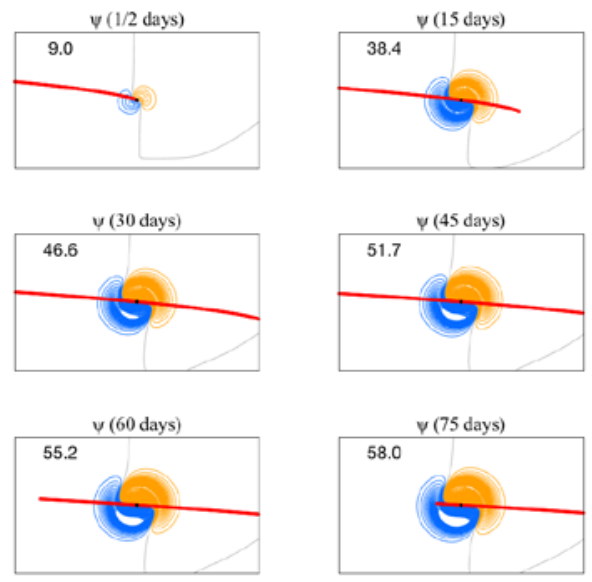

F i g. 1. Development of the beta-gyres in the linear model (18) - (20) for a point SV in the reference frame attached to the vortex (black dot in the center). Blue streamlines are negative, orange ones positive; thick red line shows the vortex trajectory. The vortex intensity $A=11 A_{0}$ (see Table 1); numbers in the upper left corner indicate the maximum values of $\psi$; contour interval $\mathrm{CI}=2$. The size of the subdomain shown is $1200 \times 700$ with the resolution of $25 \mathrm{~km}$

As a result, a symmetric dipole that changes in time and space develops in the vicinity of the SV; this dipole (the beta-gyres), centered at the SV, is what sets the $\mathrm{SV}$ into motion, the anticyclonic (cyclonic) beta-gyre being always located to the northeast (southwest) of the SV. At the very beginning, the dipole axis is directed nearly along the meridian, but it turns counterclockwise with time, resulting in the entire system moving to the northwest.

The approximate equation (17a) and, accordingly, the solutions (18), (19) and (20) are applicable at small times only, since the spatial derivatives of the vorticity $\Omega$ grow linearly with $t$, and the terms discarded in (16) eventually become, at some time, as large as the others. To estimate this time, let us non-dimensionalize the equation (16), using the period $T_{v}=2 \pi p^{-2} / A$ of the revolution of a fluid parcel around the center of the SV as the time scale, and the SV size $p^{-1}$ as the length scale. The scale of the regular streamfunction $\psi$, equal to $\Psi=\beta / p \max \left(p^{2}, a^{2}\right)$, is found from the condition that the first three terms in (16) are of the same order of magnitude. Accordingly, due to (17b) and (17c), the characteristic translation speed of the vortex is $U_{v}=\beta / \max \left(p^{2}, a^{2}\right)$. The resulting dimensionless equation has the following form

$$
\begin{aligned}
& \partial_{t} \Omega+J\left(\psi_{s}, \Omega\right)+\partial_{x} \psi_{s}+\left(1-a^{2}\right) J\left(\psi+\dot{x}_{0} y-\dot{y}_{0} x, \psi_{s}\right)+ \\
& +\alpha\left[\psi_{x}+J\left(\psi+\dot{x}_{0} y-\dot{y}_{0} x, \Omega\right)\right]=0 ;
\end{aligned}
$$

while the conditions (17b), (17c), (17d) in the dimensionless form remain unchanged. In (21), $a=a / p, \psi_{s}=-K_{0}\left(\left|\mathbf{r}-\mathbf{r}_{0}\right|\right)$, the notations for $\psi, x_{0}, y_{0}, \Omega$ remain the same as before, and the parameter

$$
\alpha=2 \pi \Psi / A=2 \pi \beta / p A \max \left(p^{2}, a^{2}\right)=T_{v} / T_{w},
$$


where $T_{w}=\max \left(p^{2}, a^{2}\right) / \beta p$ is the so-called "wave" scale, equal to the characteristic time during which the vortex travels the distance equal to its size $p^{-1}$. As already mentioned, the second term in square brackets in (21) grows linearly with time, i.e., formally, the equation (17a) and its solution (18), (19), (20) are applicable at times $\alpha t<<1$, or, in the dimensional form, at $t<<T_{w}$. Numerical experiments with the full equations (7) and (8), however, demonstrate a much longer-term applicability of the linear solution.

\section{Numerical algorithm for calculating systems of singular vortices}

An obvious difficulty of numerically integrating systems (4), (5) and (7), (8) is associated with the singularity of the function $\psi_{s}$. There are two ways to work around this problem. The first one consists in replacing the Bessel vortex in the finite-difference representation of the problem (7), (8) by a similar intense regular vortex and carrying out the integration with sufficiently small steps in space and time, which ensure the stability and convergence of the scheme. The scheme's quality is estimated by using the conservation integrals (10) - (14) obtained above and also by comparing the numerical solution with the analytical solution of the linear problem (17), which is close to the solution of the full problem at sufficiently small times. When choosing a "finite-difference SV", it is natural to proceed from the fact that the singular function in (6) is the solution to the Helmholtz problem: $\nabla^{2} \psi_{s}-p^{2} \psi_{s}=A \delta\left(x-x_{0}\right) \delta\left(y-y_{0}\right)$, and to replace $\psi_{s}$ by the solution $\psi_{s}^{*}$ of the finite-difference analogue of this equation:

$$
\left[\psi_{s_{i, j+1}}^{*}+\psi_{s_{i, j-1}}^{*}+\psi_{s_{i+1, j}}^{*}+\psi_{s_{i-1, j}}^{*}-\left(4+p^{2} \Delta^{2}\right) \psi_{s_{i, j}}^{*}\right] / \Delta^{2}=A \delta_{i, i 0} \delta_{j, j 0} / \Delta^{2} .
$$

Here $\Delta$ is a grid size; $\psi_{s_{i, j}}^{*}$ is the singular streamfunction at the grid point $(i, j)$; the grid point $(i 0, j 0)$ is the center of the singular vortex and $\delta_{i, j}$ is the Kronecker delta, which is equal to 1 at $i=j$ and is zero otherwise.

In Fig. 2, the exact expression (6) for the point vortex $(p=a)$ interpolated to the spatial grid for $R_{d}=600 \mathrm{~km}$ is compared with the numerical solution of the equation (23) at four different spatial resolutions $\Delta=25,50,100,200 \mathrm{~km}$. The graphs are given in dimensionless form with the scale for the streamfunction equal to $U \Delta$, where the scale of the velocity is $U=10 \mathrm{~ms}^{-1}$. It is seen that for all resolutions the "numerical" singular streamfunction $\psi_{s}^{*}$ coincides with the exact expression $\psi_{s}$ at all the grid nodes, except for the vortex center, where $\psi_{s}^{*}$ is finite. 


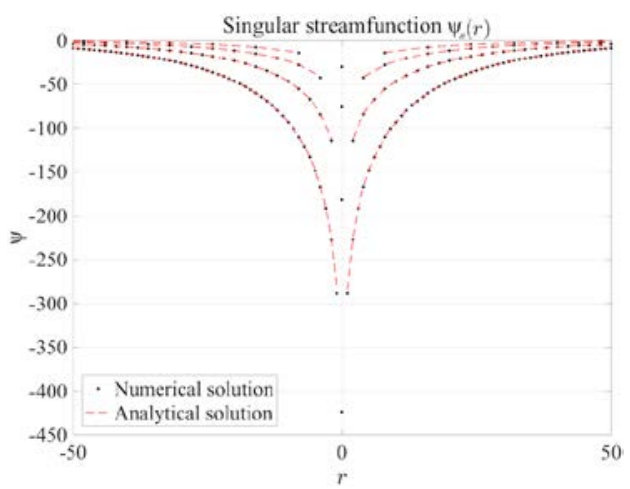

F i g. 2. Comparison of the profiles of the "numerical" singular vortex $\psi_{s}^{*}$ with the exact analytical solution $\psi_{s}$ for the point SV

In the finite-difference model, the corresponding singular streamfunction $\psi_{s}^{*}$ is interpolated by cubic splines to the grid nodes in the close vicinity ( \pm 50 grid points) of the SV vortex's center $\mathbf{r}_{0}(t)$ (which does not necessarily coincide with any grid node); outside of this area, $\psi_{s}^{*}$ is calculated by the exact formula (6). In addition, cubic splines are used to calculate the SV velocity $\left(\dot{x}_{0}, \dot{y}_{0}\right)$ in terms of the spatial derivatives of the regular streamfunction $\psi$ in (8a) and (8b). Thus, knowing the SV position $\mathbf{r}_{0}(t)$ and the regular streamfunction $\psi$ at the moment $t$, the singular streamfunction $\psi_{s}^{*}$ at each grid node and the SV's velocity $\left(\dot{x}_{0}, \dot{y}_{0}\right)$ can be calculated. This allows one to find, from (7), the regular potential vorticity $\nabla^{2} \psi-a^{2} \psi$ and, accordingly, the regular streamfunction $\psi$ and the new vortex position from (8a) and (8b) at the next time step.

For this purpose, equation (7) is approximated in a finite-difference form on a uniform grid in an $x$-periodic zonal channel of the length $L_{x}$ and width $L_{y}$. To represent the spatial derivatives, the second-order central differences are used; integration in time is carried out using the "leapfrog" scheme. On the zonal boundaries of the channel, no-flow and no slip conditions $\left(\psi_{x}=\psi_{y}=\psi_{y y y}=0\right)$ are utilized, the advective terms are represented using the fourth-order Arakawa scheme [18]; the mass and momentum constraints are also enforced [19]. The channel width $L_{y}$ is chosen to be large enough so that the streamfunction and its derivatives remain small near the zonal boundaries. In addition, the duration $T$ of each numerical experiment was chosen not to exceed the time required for the fastest Rossby wave to travel the distance $L_{x}: T \sim L_{x} / \beta R_{d}^{2}$. As it will be seen, the SV zonal speed does not exceed the limiting velocity of Rossby waves $\beta R_{d}^{2}$; therefore, the above restriction on $T$ ensures that the vortex is not affected by Rossby waves emitted by the SV images outside of the channel. With these constraints, the solutions of the channel model approximate well the SV evolution on an infinite beta-plane. 


\section{Model parameters used in the numerical experiments}

\begin{tabular}{|c|c|}
\hline Parameter & Parameter description \\
\hline$L_{x}=51200 \mathrm{~km}$ & Channel length \\
\hline$L_{y}=30000 \mathrm{~km}$ & Channel width \\
\hline$T=80$ days & Experiment duration \\
\hline$\beta=2 \cdot 10^{-11} \mathrm{~m}^{-1} \cdot \mathrm{s}^{-1}$ & Latitudinal derivative of the Coriolis parameter \\
\hline$R_{d}=600 \mathrm{~km}$ & Rossby deformation radius \\
\hline$A=m A_{0}, A_{0}=2 \pi \beta R_{d}^{3}, m=1,3,5,7,9,11$ & Vortex intensity \\
\hline $300 \mathrm{~km}$ & Vortex size: small SV \\
\hline $600 \mathrm{~km}$ & Vortex size: point SV \\
\hline $1200 \mathrm{~km}$ & Vortex size: large SV \\
\hline$a=R_{d}^{-1}$ & Inverse Rossby radius \\
\hline$p=L_{v}^{-1}$ & Inverse size of the SV \\
\hline$\Delta_{x}=\Delta_{y}=\Delta=25 \mathrm{~km}$ & Spatial grid size \\
\hline$\Delta t=30 \mathrm{~s}$ & Timestep \\
\hline$K=2 \cdot 10^{13} \mathrm{~m}^{4} \cdot \mathrm{s}^{-1}$ & Hyperviscosity \\
\hline
\end{tabular}

In the second method for solving the problem of singular coefficients in the systems (4), (5) and (7), (8), the Lagrangian integral (14) is used. Knowing the vortex position at time $t$, it is possible to determine the value of the regular vorticity $\Omega$ at the center of the vortex without solving equations (4), (7), and use this value when to compute the regular streamfunction near the center of the SV. This numerical algorithm was also implemented (see [10] and Section 5 below); the numerical results obtained by using these two different algorithms turned out to be very close.

To study the dynamics of an isolated monopole on the beta-plane, a number of numerical experiments were carried out, with parameters summarized in the table. The parameters chosen are typical for the eddies in the mid-latitude atmosphere, but all the results are simply recalculated for the case of the ocean by decreasing the spatial scales $L_{x}, L_{y}, R_{d}, L_{v}, \Delta$ by a factor of 10 , the characteristic velocities by a factor of 100 , and increasing the time scale by a factor of 10 .

\section{Results of numerical experiments}

The solution of the problem (21), (17b), (17c), (17d) depends on two dimensionless parameters $\alpha$ and $\bar{a}$, which completely determine the regime of the SV evolution. If the horizontal scale of the vortex $L_{v}=p^{-1}$ is fixed, then this 
evolution is determined the single parameter $\alpha$, i.e., by the ratio $\beta$ / $A$. Here we only consider the case of a point vortex (cyclone), for which the solution (18) (20) is also an exact solution of problem (21), (17b), (17c), (17d) at $\alpha=0$, i.e., in the limit of an infinitely large SV intensity $A$, small and large vortices (see table) behave in the same way (see also [10]).

Large intensity $A$. The evolution of the vortex, computed by using the full equations (7), (8) in the case of the large intensity $A=11 A_{0}$ (see table) is shown in Fig. 3. Similar to the linear regime shown in Fig. 1, the singular cyclone moves to the northwest, but the field $\psi$ has a much more complex structure. In the vicinity of the vortex, the $\beta$-gyres appear again, their intensity increasing with time, but they quickly cease to be symmetric and, starting from about $t=3.5$ day, the intensity of the cyclonic $\beta$-gyre stops to grow.

The singular vortex gradually drifts into the $\beta$-gyre of the opposite sign (that is, into the anticyclonic beta-gyre for a cyclonic SV), which cannot happen in the "purely" linear regime (see Fig. 1). The dispersion term $\beta \psi_{x}$ in (7) is responsible for the far-field radiation of Rossby waves, and a wave tail appears to the east of the vortex (a qualitative description of this process can be found, for example, in [10] and [20]). Nevertheless, the tail amplitude remains small, at least for the calculated times (here, up to $t=80$ days).
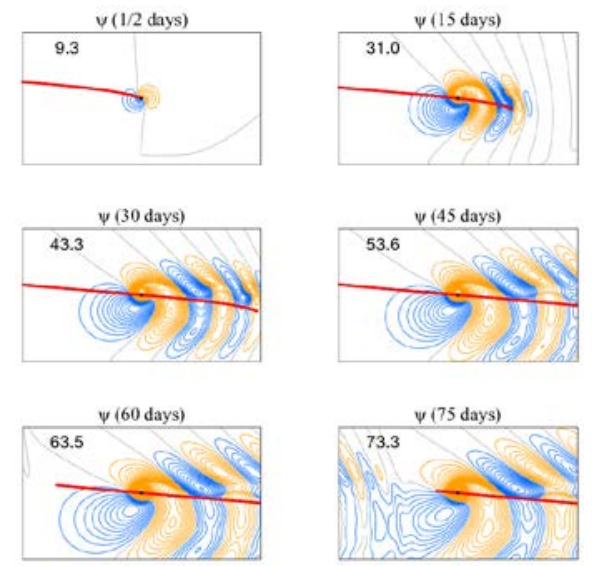

F i g. 3. Evolution of the regular streamfunction $\psi$ computed using the full system of equations. Thin gray contours show zero streamlines; other notations and parameters are the same as in Fig. 1

It is important that these changes are not accompanied by the loss of the connection between the vortex and the cyclonic $\beta$-gyre, as is the case during the nonlinear stage of the flow development (see [10] and below), and the resulting velocities and trajectory of the vortex are close to those calculated using the linear model (17) (see Fig. 4). We can thus call the evolution regime at large intensities $A$ (or, equivalently, at small $\beta$ ) quasilinear. 

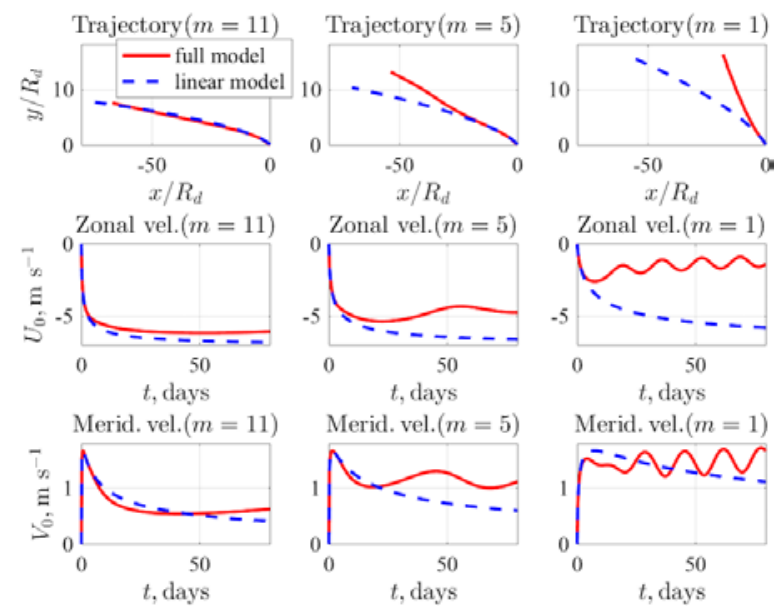

F i g. 4. Trajectories and velocities of the point vortex for various values of $A$. Left column: $A=11 A_{0}$; middle column: $A=5 A_{0}$; right column: $A=A_{0}$

Small intensity $A$. With a decrease in the intensity of the vortex $A$ (and/or with an increase in $\beta$ ), the evolution of the vortex becomes more complicated: a relatively short quasilinear stage is replaced by a nonlinear one, when, in (7), the dispersion term $\beta \psi_{x}$ and self-interactions of the regular component $J(\psi, Q)$ become important. A typical example of this evolution is shown in Fig. 5 for the case $A=A_{0}$. At first, up to about $t=10$ day, the evolution only slightly deviates from the quasilinear regime in Figs. 1, 3: the regular field consists mainly of a dipole in the vicinity of the vortex and the waves, which follow the vortex, but do not significantly affect the motion of the vortex. The same conclusion can be drawn from the velocity graphs in Fig. 4 for $A=A_{0}$ : up to about $t=10$, the SV velocity components are close to "linear". At the same time, the intensity of the anticyclonic $\beta$-gyre and that of the wave field at $A=A_{0}$ grow (in relative units) much faster than in the case of the large-intensity SV with $A=11 A_{0}$. This growth is accompanied by a faster and deeper penetration of the SV into the anticyclonic $\beta$-gyre. In this case, the intensity of the cyclonic $\beta$-gyre, after the initial relaxation period, practically does not increase, remaining at approximately constant level, which is much lower than the intensity of the anticyclonic $\beta$-gyre at sufficiently long times.

After $t=10$ days, the character of the SV evolution changes dramatically. Both components of the vortex velocity cease to change monotonically with time and instead begin to oscillate around some mean levels (Fig. 4). The average level of the zonal velocity gradually decreases in magnitude, i.e., the vortex drift to the west gradually slowing down with time. The meridional velocity of the vortex, after a sharp increase and then a decrease at the linear stage, rapidly increases again, oscillating around the value of about $1.5 \mathrm{~ms}^{-1}$ (Fig. 4). Accordingly, the vortex moves fairly quickly along the meridian to the north. Thus, for $t>10$ days, the solution already strongly deviates from the linear regime (17) - (20), which is why we called this stage nonlinear. 

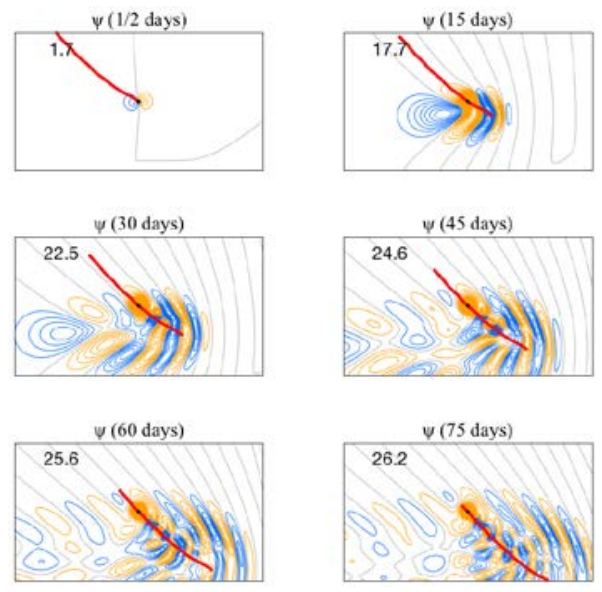

F i g. 5. The same as in Fig. 3 but for $A=A_{0}$. Contour interval CI=0.5

The streamfunction field for $t>10$ days is also substantially modified in comparison with that in the quasilinear regime (compare Figs. 1, 3, and 5). The vortex continues to move inside the anticyclonic $\beta$-gyre and eventually finds itself in the immediate vicinity of the center of this gyre (see Fig. 6). The anticyclonic beta gyre and the vortex "inside" of it make up a vortex pair drifting to the northwest. As the vortex approaches the center of the anticyclonic beta-gyre, the intensity of the latter increases rapidly; at the same time, this betagyre becomes more compact, acquiring an almost circular shape. Meanwhile, the magnitude of the cyclonic beta-gyre, starting from a certain moment, ceases to grow and turns out to be much smaller than the intensity of the anticyclonic betagyre. Over time, the cyclonic beta-gyre "spreads out" and eventually loses its direct connection with the singular vortex (see Fig. 5).
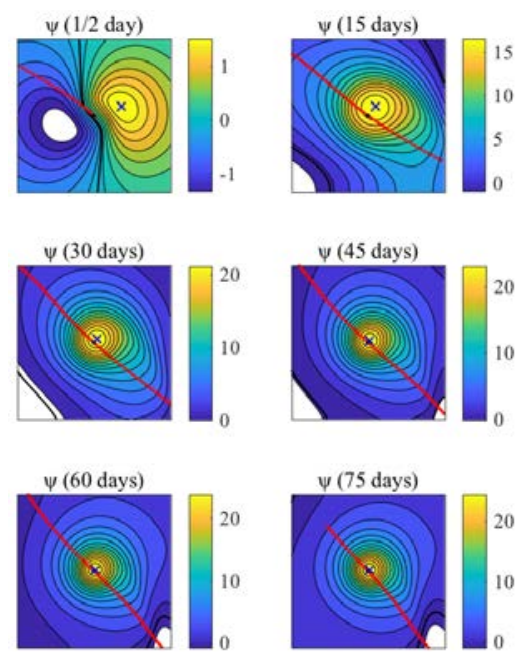

F i g. 6. The drift of a singular cyclone towards the center of the anticyclonic $\beta$-gyre. The dot denotes the center of the singular cyclone; the $x$-symbol - the center of the $\beta$-gyre; red curves trace the vortex trajectory; $p=a, A=A_{0}$. The size of the sub-domain shown is $50 \times 50$, i. e., approximately, $2 \times 2$ Rossby radii 
Another typical difference between the nonlinear regime and the quasilinear regime is that the Rossby waves radiated by the vortex cease to "obediently" follow it (as, for example, in Figs. 3 and 5 at $t=15$ days) and begin to interact with the vortex (Fig. 5, $t=30$ days and further). It is this interaction that explains the appearance of the fluctuations in the velocity graphs in Fig. 4; a simple analysis of this and other nonlinear regimes shows that the number of the vortex's zonalvelocity maxima always coincides with the number of Rossby wave crests that the vortex crosses during its evolution.

An important conclusion that can be drawn from Fig. 4 is that the linear regime describes well the motion of the vortex, especially its trajectory, at times significantly exceeding the formal time of applicability of the linear equations (17). In Section 3, we showed that formally this time does not exceed the wave scale $T_{w}=\max \left(p^{2}, a^{2}\right) / \beta p$ equal to $1 / \beta R_{d}$ for the point vortex. For our parameters $T_{w}=1 / \beta R_{d} \approx 1$ day and from Fig. 4 , it follows that the actual time of applicability is much longer than $T_{w}$, but strongly depends on the intensity of the vortex $A$ : the larger $A$, the longer the linear theory works. At a large intensity ( $A=11 A_{0}$ ) the vortex velocities are close to linear, and the trajectories in the linear case and complete theory practically coincide throughout the entire computation time. At an average intensity $\left(A=5 A_{0}\right)$, the difference in velocity increases, but the trajectory remains close to linear, at least up to $t=36$ day (the dots on the trajectory graphs are separated by 3 days). Even in the case of a vortex of small intensity $\left(A=A_{0}\right.$ ) the trajectory is close to linear for at least 15 days, which is more than an order of magnitude longer than $T_{w}$.

Integrals of motion. The evolution of the integrals of motion obtained in Section 2 is shown in Fig. 7 for different vortex intensities. Friction does not affect the momentum integral, which is well conserved in all cases. The same is true for the energy integral, which is only weakly affected by friction. It can be seen that the terms $E_{1},-\left.A \psi\right|_{\mathbf{r}=\mathbf{r}_{0}(t)}$ in (10) have a similar magnitude, while the magnitude of their sum is much less. Comparison of this sum with the time-integrated dissipative term $D_{1}+D_{2}$ leads to the conclusion that it is indeed the dissipation that is responsible for the "non-conservation" of energy, as follows from equation (10a).

Friction has the larger effect on the enstrophy $S_{1}-\beta A y_{0}$, which noticeably decreases over time in all cases. According to (11a), the decay of the sum $S_{1}-\beta A y_{0}$ must be due to the time-integrated dissipative term $D_{3}$, which is observed in all cases.

Some of the qualitative features of the behavior of a singular monopole can be interpreted in terms of the above conservation integrals. For example, in the absence of friction (i.e. at $D_{3}=0$ ), it follows from (11a) that the growth of regular enstrophy $S_{1}$ (which does take place in all cases; see Fig. 7) should lead to a monotonic drift of the singular cyclone $(A>0)$ to the north, since this growth should be compensated by an increase in the value of $-S_{2}=\beta A y_{0}$ in (11a). 
Similarly, the merger of the cyclone with the anticyclonic $\beta$-gyre can be derived from the conservation of energy. For example, from (10a) it follows that for a point vortex in the absence of friction

$$
\left.\partial_{t} \psi\right|_{\mathbf{r}=\mathbf{r}_{0}(t)}=\partial_{t} E_{1} / A>0,
$$

since the energy of the regular component $E_{1}$ increases monotonically, as can be seen from Fig. 7. Therefore, the value of $\left.\psi\right|_{\mathbf{r}=\mathbf{r}_{0}(t)}$ increases with time, i.e., the singular vortex drifts from the axis of the $\beta$-gyres dipole (where $\psi=0$ ) to the region of positive values $\psi$ - hence, to the anticyclonic $\beta$-gyre. The same is true for large and small vortices, since in both cases the sum $E_{1}+E_{2}$ increases with $t$ (not shown here).

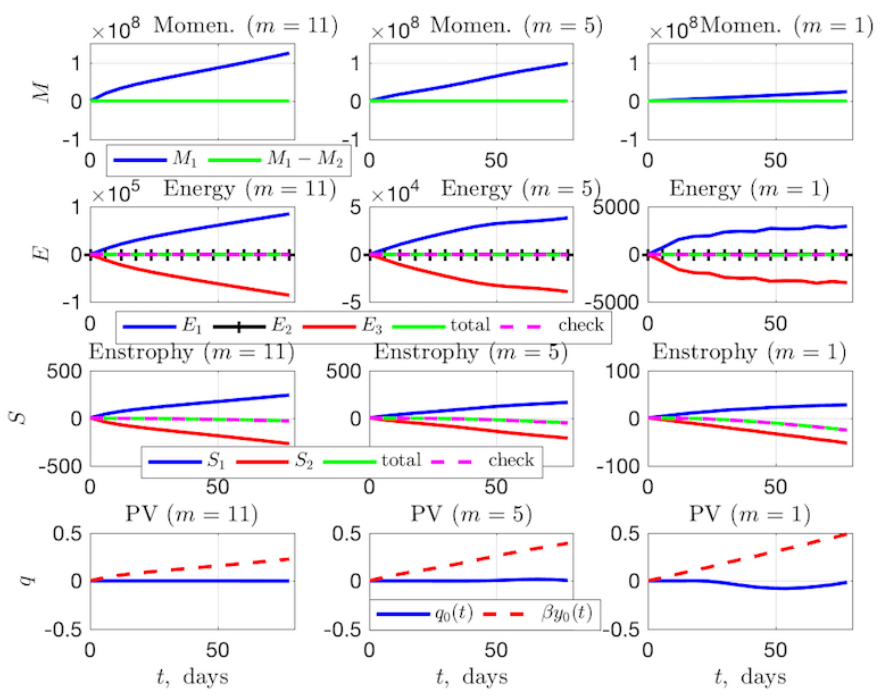

F i g. 7. Integrals of motion for the singular vortices of different intensity. Top row: zonal momentum; second row: energy; third row: enstrophy; bottom row: potential vorticity

The conservation of momentum, energy, and enstrophy indicates that the numerical scheme developed here approximates the solution of the original problem with a sufficient degree of accuracy. The Lagrangian characteristic the potential vorticity $q$ of the regular field at the center of the vortex (see (14)) is nearly conserved for the vortex of large intensity with $m=11$; this conservation is worse for $m=5$ and the worst - for $m=1$. In principle, this is understandable, since the relation (14) is strictly obtained only for a "truly" singular vortex with a $\delta$-function-like vorticity. The "finite-difference" singular vortex used by us obviously differs from the "true" one, and this difference increases with the decrease of $m$. Nevertheless, an important question remains: How does the poor "conservation" of $q$ affect the trajectory and velocities of the vortex?

For verification, we computed the evolution of the vortex using the alternative numerical scheme mentioned above in Section 4. In this scheme, the conservation of potential vorticity (14) at the center of the vortex is hardwired; the remaining 
problem is that, in the absence of viscosity, there is a strong variability of the PV in the near vicinity of the vortex [2], which does not allow one to compute the PV with the sufficient accuracy at the grid nodes close to the center of the vortex. At the same time, even the minimum viscosity leads to almost instantaneous homogenization of the PV in the close vicinity of the SV (see Fig. 8). This homogenization has practically no effect on the regular component, but it allows us to develop a modified numerical algorithm. In this algorithm, the regular vorticity $\Omega$ at the center of the vortex is determined, at each time step, from (14), and that at the four nearest nodes - from the known value of the homogenized potential vorticity. Obviously, with this approach, there is no difference between the "exact" singular vortex (6) and its finite-difference analog, since the location of singularity is never involved in approximating the derivatives of the flow fields. The important result is that the numerical solutions in both approaches are very close to each other, i.e., the variations of the regular PV near the center of the vortex do not practically affect either the structure of the regular field or the velocity and trajectory of the SV.
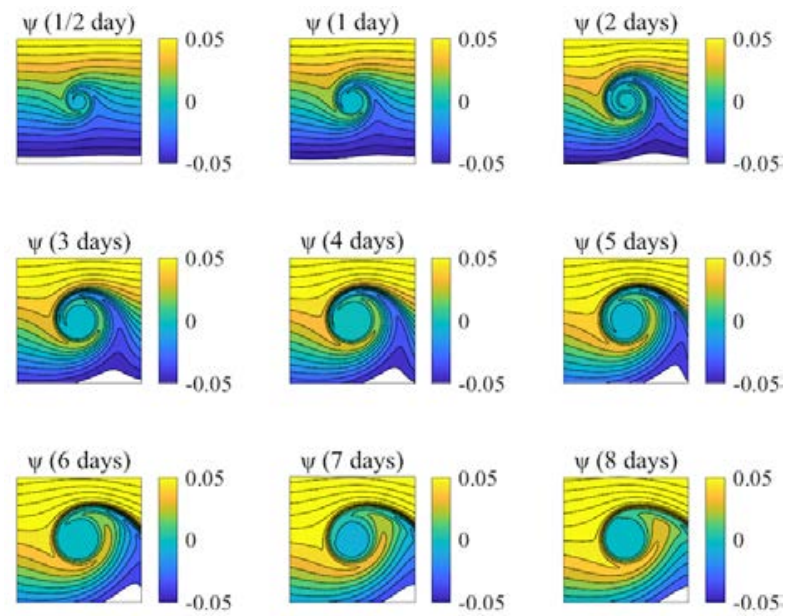

F i g. 8. Evolution of the total potential vorticity $\nabla^{2} \psi-a^{2} \psi+\beta y$ for the point SV at initial stage. The isolines of potential vorticity are shown in the $50 \times 50$ subdomain with the origin at the center of the vortex

\section{Discussion of the results}

We have presented a brief overview of the theory of the singular vortices on a beta-plane, first proposed in [2]. The main emphasis was on recent numerical studies of the evolution of an individual singular monopole interacting with the regular flow it generates [10]. Two numerical algorithms have been developed and implemented to compute such an evolution. In the first of these algorithms, the "theoretical" singular vortex is replaced by an equivalent "numerical" regular vortex, compatible with the finite-difference formulation. Another algorithm uses the Lagrangian conservation of the regular potential vorticity at the center of the vortex and homogenization of the PV in its vicinity created by an arbitrarily small friction. Both algorithms give practically identical results, demonstrating that the proposed model, firstly, accurately reproduces the initial quasilinear stage of 
the SV evolution (which is calculated analytically for the point SV) and, secondly, preserves several invariants of motion derived from the original continuous equations.

The vortex evolution consists of two stages (without loss of generality, we will discuss the case of a singular cyclone). At the first (quasilinear) stage, the SV motion to the northwest is induced by a secondary dipole in the vicinity of the SV (the so-called $\beta$-gyres), generated via the near-field radiation of Rossby waves by the SV. In this case, the zonal velocity of the vortex rapidly decreases from zero to a certain negative minimum value; meanwhile, the meridional velocity at first rapidly increases, and then just as quickly drops to its minimum (but still positive) value. The duration of the quasilinear stage strongly depends on the SV intensity: an increase (decrease) in the intensity prolongs (shortens) this stage and deepens (increases the magnitude) of the velocity minima.

The quasilinear stage is gradually replaced by a nonlinear stage, during which the dipolar structure of the regular field in the vicinity of the SV is destroyed, the cyclonic $\beta$-gyre loses its connection with the SV and gradually disappears. The singular vortex merges with the anticyclonic $\beta$-gyre, together forming a compact vortex pair, which continues to move northwestward and interacts with the Rossby waves emitted by the vortex. This interaction leads to oscillations of the vortex velocity components, the magnitude of SV zonal velocity (directed to the west) decreasing, while its meridional velocity remaining at a constant level. With decreasing SV intensity, its zonal velocity, on average, decreases in magnitude, and its meridional velocity increases; accordingly, the SV trajectory bends towards the pole. The nonlinear stage of the SV evolution on a beta-plane was first presented in our work [10].

In this study, we only presented the results for the point vortex with $p=a$; the evolution of large and small vortices with $p \neq a$ is qualitatively similar, with some quantitative differences described in [10].

\section{REFERENCES}

1. Kamenkovich, V.M., Koshlyakov, M.N. and Monin, A.S., 1987. Synoptical Eddies in the Ocean. Leningrad: Gidrometeoizdat, 510 p. (in Russian).

2. Reznik, G.M., 1992. Dynamics of Singular Vortices on a Beta-Plane. Journal of Fluid Mechanics, 240, pp. 405-432. doi:10.1017/S0022112092000144

3. Reznik, G.M. and Dewar, W.K., 1994. An Analytical Theory of Distributed Axisymmetric Barotropic Vortices on the $\beta$-Plane. Journal of Fluid Mechanics, 269, pp. 301-321. https://doi.org/10.1017/S0022112094001576

4. Sutyrin, G.G. and Flierl, G.R., 1994. Intense Vortex Motion on the Beta Plane: Development of the Beta Gyres. Journal of the Atmospheric Sciences, 51(5), pp. 773-790. https://doi.org/10.1175/1520-0469(1994)051<0773:IVMOTB>2.0.CO;2

5. Llevellyn Smith, S.G., 1997. The Motion of a Non-isolated Vortex on the Beta-Plane. Journal of Fluid Mechanics, 346, pp. 149-179. https://doi.org/10.1017/S0022112097006290

6. Sutyrin, G., Hesthaven, J., Lynov, J. and Rasmussen, J., 1994. Dynamical Properties of Vortical Structures on the Beta-plane. Journal of Fluid Mechanics, 268, pp. 103-131. doi:10.1017/S002211209400128X

7. Lam, J.S-L. and Dritschel, D.G., 2001. On the Beta-Drift of an Initially Circular Vortex Patch. Journal of Fluid Mechanics, 436, pp. 107-129. https://doi.org/10.1017/S0022112001003974 
8. Korotaev, G.K. and Fedotov, A.B., 1994. Dynamics of an Isolated Barotropic Eddy on a Betaplane. Journal of Fluid Mechanics, 264, pp. 277-301. https://doi.org/10.1017/S0022112094000662

9. Early, J.J., Samelson, R.M. and Chelton, D.B., 2011. The Evolution and Propagation of Quasigeostrophic Ocean Eddies. Journal of Physical Oceanography, 41(8), pp. 1535-1555. https://doi.org/10.1175/2011JPO4601.1

10. Kravtsov, S. and Reznik, G., 2019. Numerical Solutions of the Singular Vortex Problem. Physics of Fluids, 31, 066602. https://doi.org/10.1063/ 1.5099896

11. Firing, E. and Beardsley, R.C., 1976. The Behavior of a Barotropic Eddy on a $\beta$-Plane. Journal of Physical Oceanography, 6(1), pp. 57-65. https://doi.org/10.1175/15200485(1976)006<0057:TBOABE >2.0.CO;2

12. Carnevale, G.F., Kloosterziel, R.C. and Van Heijst, G.J.F., 1991. Propagation of Barotropic Vortices over Topography in a Rotating Tank. Journal of Fluid Mechanics, 233, pp. 119-139. https://doi.org/10.1017/S0022112091000411

13. Saffman, P.G., 1993. Vortex Dynamics. Cambridge: Cambridge University Press, 311 p. https://doi.org/10.1017/CBO9780511624063

14. Reznik, G.M. and Kizner, Z., 2007. Two-Layer Quasi-Geostrophic Singular Vortices Embedded in a Regular Flow. Part 1. Invariants of Motion and Stability of Vortex Pairs. Journal of Fluid Mechanics, 584, pp. 185-202. https://doi.org/10.1017/S0022112007006386

15. Reznik, G. and Kizner, Z., 2007. Two-Layer Quasi-Geostrophic Singular Vortices Embedded in a Regular Flow. Part 2. Steady and Unsteady Drift of Individual Vortices on a Beta-Plane. Journal of Fluid Mechanics, 584, pp. 203-223. doi:10.1017/S0022112007006404

16. Klyatskin, K.V. and Reznik, G.M., 1989. Point Vortices on a Rotating Sphere. Oceanology, 29(1), pp. 12-16.

17. Reznik, G.M. and Kravtsov, S.V., 1996. The Dynamics of a Barotropic Singular Vortex on the Beta Plane. Izvestiya, Atmospheric and Oceanic Physics, 32(6), pp. 701-707.

18. Arakawa, A., 1966. Computational Design for Long-Term Numerical Integration of the Equations of Fluid Motion: Two-Dimensional Incompressible Flow. Part I. Journal of Computational Physics, 1(1), pp. 119-143. http://dx.doi.org/10.1016/0021-9991(66)90015-5

19. McWilliams, J.C., 1977. A Note on a Consistent Quasigeostrophic Model in a Multiply Connected Domain. Dynamics of Atmospheres and Oceans, 1(5), pp. 427-441. https://doi.org/10.1016/0377-0265(77)90002-1

20. Reznik, G.M., 2010. Dynamics of Localized Vortices on the Beta Plane. Izvestiya, $\begin{array}{lllll}\text { Atmospheric and Oceanic Physics, 46(6), pp. 784-797. } & \end{array}$ https://doi.org/10.1134/S0001433810060095

About the authors:

Grigory M. Reznik, Chief Research Associate, Shirshov Institute of Oceanology, Russian Academy of Sciences (36, Nakhimovskiy Ave., Moscow, 117997, Russian Federation), Dr. Sci. (Phys.-Math.), ORCID ID: 0000-0003-2626-2239, ResearcherID: W-3112-2017, greznikmd@yahoo.com

Sergey V. Kravtsov, Professor, Department of Mathematical Sciences, University of Wisconsin-Milwaukee (P. O. Box 413, Milwaukee, WI 53201, USA), Ph. D. (Phys.-Math.), ORCID ID: 0000-0003-3653-9596, ResearcherID: ABA-2061-2020, kravtsov@uwm.edu

Contribution of the co-authors:

Grigory M. Reznik - statement of the problem, theoretical part of the work, interpretation of the numerical experiments, writing the paper

Sergey V. Kravtsov - realization of numerical algorithm, implementation and interpretation of numerical experiments, writing the paper

All the authors have read and approved the final manuscript.

The authors declare that they have no conflict of interest. 